\title{
Pathology of esophageal cancer and Barrett's esophagus
}

\author{
Shilpa Jain*, Sadhna Dhingra* \\ Department of Pathology and Immunology, Baylor College of Medicine, Houston, TX 77030, USA \\ *These authors contributed equally to this work. \\ Correspondence to: Sadhna Dhingra, MD. Assistant Professor, Department of Pathology and Immunology, Baylor College of Medicine, Y144, Baylor \\ St. Luke's Medical Center, 6720, Bertner Avenue, Houston, TX 77030, USA. Email: sadhna.dhingra@bcm.edu.
}

\begin{abstract}
Esophageal cancer is a serious malignancy with high mortality. The two common distinctive pathologic subtypes of esophageal cancer are squamous cell carcinoma and adenocarcinoma. These differ with regards to etiology, ethnic distribution, pathogenesis, and location in the esophagus. The precursor lesions are also unique to each subtype. Squamous cell carcinoma is more common in East Asia, is linked to smoking and tobacco use, more commonly involves the middle esophagus, and the precursor lesion is squamous dysplasia. Adenocarcinoma is more common in the United States and certain European countries, associated with obesity and gastroesophageal reflux disease (GERD), more commonly involves the distal esophagus, and the precursor lesion is Barrett's esophagus. Endoscopic surveillance with biopsy evaluation is the standard of care in high-risk groups. Endoscopic ablative therapies for early cancers have lower morbidity than surgery. Despite increased awareness, identification of high-risk groups and endoscopic surveillance, a large proportion of patients present with advanced cancers. Surgery and chemoradiation, either in neo-adjuvant or adjuvant setting, is the usual treatment for patients with advanced but resectable esophageal cancers. The prognosis and further management largely depends upon the pathologic tumor-node-metastasis (TNM) staging provided by the American Joint Committee on Cancer (AJCC) and the International Union against Cancer. Currently, the 7th edition of TNM staging system is being applied for prognostication and this is more focused on pathologic evaluation. Eighth edition of AJCC/UICC TNM staging has been introduced and will be implemented for clinical use in 2018.
\end{abstract}

Keywords: Esophagus; squamous cell carcinoma; adenocarcinoma; Barrett's esophagus; carcinoma

Submitted Nov 02, 2016. Accepted for publication Jan 18, 2017.

doi: $10.21037 /$ acs.2017.03.06

View this article at: http://dx.doi.org/10.21037/acs.2017.03.06

\section{Introduction}

Primary esophageal cancer comprise majority (greater than 95\%) of all esophageal malignancies. Other malignant tumors such as lymphoma, sarcoma or metastasis are rare in the esophagus. Esophageal cancer represents $1 \%$ of all new cancer cases in the United States (SEER) (1). It is an aggressive cancer with a high mortality and an average 5 -year survival of $18.4 \%$ (1). Worldwide, this is the eighth most common incident cancer, and sixth most common cause of mortality (2). Esophageal cancer is a disease of older age with a peak incidence in 6th to 7th decade. Histologically, there are two predominant types of primary esophageal cancers: squamous cell carcinoma and adenocarcinoma.
Both of these are distinct entities with some overlap, with regards to epidemiologic distribution, risk factors, pathogenesis, and clinical and prognostic relevance. Esophageal squamous cell carcinoma (ESCC) is the most common esophageal cancer worldwide. The highest rates are seen in the Asian/Eastern countries. However, the epidemiology of esophageal cancer in the US, Australia and certain parts of Europe has changed over last few decades. Until the 1970s, esophageal squamous cell carcinoma (ESCC) was the most common cancer in the United States and Western Europe. Over the last few decades the rates of ESCC has declined and that of esophageal adenocarcinoma (EAC) has increased in the Western world (3). The decrease in ESCC is probably due to decrease in alcohol 
and tobacco abuse, while the increase in EAC is linked to increase in obesity and GERD. In the United States, ESCC occurs more commonly in African Americans and white women, whereas EAC shows a strong male predominance and occurs more commonly in white males (2). This review article will focus on the histopathologic aspects of esophageal squamous cell carcinoma, Barrett's esophagus and esophageal adenocarcinoma that arise in the setting of Barrett's esophagus.

\section{Esophageal squamous cell carcinoma}

ESCC most commonly occurs in the middle third of the esophagus and less commonly in the lower third. It is uncommon in the upper esophagus. Clinically, these present late and the usual presenting symptoms are dysphagia and weight loss followed by anorexia. ESCC arise from the squamous lining of the esophagus through progression of premalignant precursor lesions that occur in the presence of risk factors that cause chronic irritation and inflammation. Smoking, chewing tobacco and alcohol consumption are well-documented risk factors independently associated with ESCC $(4,5)$. Dietary factors such as a diet low in fruits and vegetables leading to low antioxidant levels and vitamin deficiencies also contribute to development of ESCC (6). The role of human papilloma virus (HPV) in development of ESCC is not well established yet (7). Certain genetic factors also play a role in development of ESCC. Nonepidermolytic palmoplantar keratoderma (Tylosis) is an autosomal dominant disease, characterized by palmer and plantar hyperkeratosis and is associated with very high frequency ESCC. These patients have a mutation in RHBDF2 located on chromosome 17q25 [tylosis esophageal cancer (TOC) gene] (8). Achalasia, a functional disorder of esophageal motility, is a known cause of ESCC due to effects of chronic food stasis and a persistent inflammatory state (9).

\section{Premalignant/precursor lesion}

Chronic esophagitis: Early studies suggested that mild to moderate chronic esophagitis was associated with family history of esophageal cancer and other risk factors of ESCC (10). Subsequently, systematic studies with endoscopic surveillance, biopsy evaluation, and follow-up to the development of ESCC, showed that esophagitis is nonspecific, and the only true precursor lesion of ESCC is squamous dysplasia $(11,12)$.

Squamous dysplasia/Intraepithelial neoplasia: Dysplasia is defined as a disordered proliferation of cells limited to the epithelium due to genetic alterations with a predisposition to invasion and metastasis (13). Squamous dysplasia is usually asymptomatic. Endoscopically, the dysplastic mucosa may appear completely normal, or may show mucosal changes of friability, erythema, erosions, plaques and nodules. Sensitivity of endoscopic examination can be increased by use of Lugol's iodine (14). Exfoliative balloon cytology can be used to obtain esophageal cells for evaluation of dysplasia for surveillance in high-risk groups (15).

Squamous dysplasia is a histologic lesion confined to epithelium and is characterized by cytologic and architectural abnormalities. The cytologic abnormalities include nuclear enlargement, hyperchromasia, pleomorphism and increased and/or abnormal mitosis. The architectural changes include loss of polarity and lack of surface maturation. The abnormality starts from the basal layer, and based on the extent of involvement of thickness of epithelium by atypical cells, the dysplasia was traditionally graded as mild (up to one-third), moderate (up to twothirds) and severe (involving upper one-third). In 2000, the WHO adopted the term "intraepithelial neoplasia" (IEN) for dysplasia, and classified IEN in a two-tier system as lowgrade or high-grade. When less than half of thickness of epithelium is involved with atypical cells it is graded as lowgrade (Figure 1A) and when greater than half of thickness is involved it is graded as high-grade. Full thickness involvement of epithelium is also called "squamous cell carcinoma in situ" (CIS) or "noninvasive squamous cell carcinoma" in the Japanese literature (13) (Figure 1B). A special type of CIS where the atypical cells are restricted to the basal cell layer is called "basal-layer type of squamous cell carcinoma in situ" in Japan (13). However, this would be designated as low grade IEN as per Western criteria. The dysplasia may extend into esophageal submucosal glands and ducts, and may mimic stromal invasion (16). The dysplastic cells may also spread horizontally in a pagetoid manner (17). Multicentricity of dysplasia and ESCC support the concept of field effect in carcinogenesis (18). Increasing grades of dysplasia predict increased risk of ESCC $(11,12,19)$.

Mimickers of dysplasia include reactive/regenerative changes secondary to esophagitis, and effects of radiotherapy or chemotherapy. The reactive epithelium shows surface maturation and lacks nuclear crowding or abnormal mitosis. Reactive nuclei are enlarged with fine vesicular chromatin and presence of small nucleoli. Radiation induced changes include cytoplasmic eosinophilia, cytoplasmic vacuolation, and low nuclear to cytoplasmic ratio with reactive nuclear 

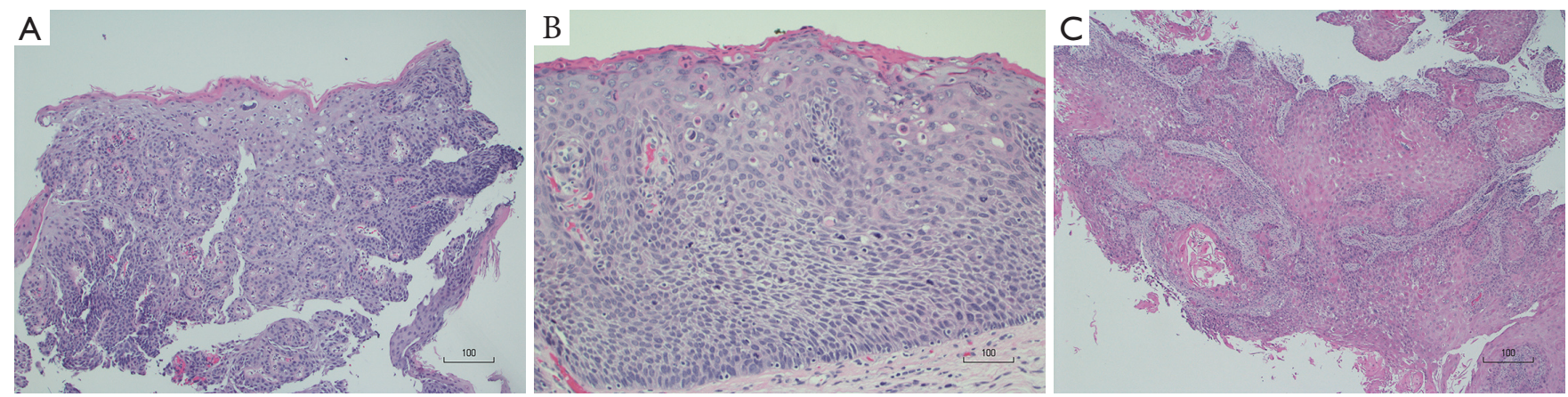

Figure 1 Squamous lesions. (A) Squamous mucosa with low grade dysplasia. Hematoxylin and Eosin stain $\times 100$; (B) squamous mucosa with high grade dysplasia/carcinoma-in-situ. Hematoxylin and Eosin stain $\times 200 ;(C)$ invasive squamous cell carcinoma. Hematoxylin and Eosin stain $\times 40$.

changes. The term "indefinite for dysplasia" can be appropriately used in uncertain cases.

\section{Invasive esophageal squamous cell carcinoma}

ESCC occurs most commonly in the middle third of esophagus followed by lower one-third and upper one-third respectively. Clinically, it presents as dysphagia, weight loss and retrosternal pain. Endoscopically, it can be polypoid, flat or ulcerated. On endoscopic ultrasound (EUS), infiltrating ESCC presents as a circumscribed diffuse wall thickening with echo-poor pattern due to destruction of layers of esophageal wall.

\section{Histopathology}

Invasion of neoplastic squamous cells into lamina propria and deeper layers defines invasive ESCC. Histologically, the tumor can show variable differentiation. Well-differentiated ESCC show presence of keratin pearls, individual cell keratinization and intercellular bridges (Figure 1C). Poorly differentiated ESCC lack these features and is determined to be squamous in origin based on pattern of infiltration, presence of IEN or in situ lesions in the adjacent squamous mucosa, or with help of immunohistochemical markers such as CK5/6 or p63. Moderately differentiated ESCC show intermediate features.

\section{Morphological variants}

Basaloid squamous cell carcinoma shows predominance of basaloid features characterized by basaloid cells with oval hyperchromatic nuclei, scant cytoplasm and solid nests with peripheral palisading. The important differential diagnosis is adenoid cystic carcinoma, which has a better prognosis.
Verrucous carcinoma is a slow growing exophytic papillary tumor, and morphologically is a very well differentiated squamous carcinoma. These pose a diagnostic challenge for pathologists, as they are papillary tumors with mild cellular atypia restricted to basal layers. These are difficult to distinguish from squamous papillomas. These tumors can have foci of invasion as a broad pushing front but have no metastatic potential. Some cases are locally infiltrative with fistula formation.

Carcinosarcoma is another histological variant with biphasic morphology composed of malignant epithelial and spindle cells, and sometimes show a mesenchymal differentiation. The spindle cells may be bland or pleomorphic with frequent mitosis. Mesenchymal differentiation may be chondroid, rhabdoid or osteoid. Immunohistochemically, the spindle cell component is cytokeratin and vimentin positive. These tumors are generally thought to have better prognosis (20), however, a recent study from Italy showed conflicting results (21).

\section{Esophageal adenocarcinoma and Barrett's esophagus}

Adenocarcinoma of the esophagus is a carcinoma with glandular differentiation that arises in the setting of Barrett's esophagus (BE). The most important etiological factor for EAC is Barrett's esophagus in the setting of gastro-esophageal reflux. Other risk factors include male sex, Caucasian race, tobacco smoking and obesity (22). Helicobacter pylori infection is inversely correlated to EAC (23).

\section{Barrett's esophagus}

Barrett's esophagus is essentially defined as columnar 
metaplasia that replaces the stratified squamous epithelium of the distal esophagus, and has a predisposition to develop adenocarcinoma (24). Diagnosis of BE involves both endoscopic and pathologic evaluation. Although the presence of nongoblet foveolar epithelium in biopsies obtained from endoscopically visible columnar-lined esophagus is sufficient for a diagnosis of Barrett's esophagus in some European countries (25), intestinal-type goblet cells (intestinal metaplasia) are required for this diagnosis in the United States (26). The lack of intestinal metaplasia (IM) is associated with lower risk of malignant transformation (27). The guidelines for diagnosis and management of $\mathrm{BE}$ are set forth by the British society of Gastroenterology (BSG) and by American Gastroenterology Association (AGA)/ American College of Gastroenterologists (AGC), and have evolved over time. As per most recent recommendation by ACG, BE should be diagnosed when there is extension of salmon-colored mucosa $\geq 1 \mathrm{~cm}$ proximal to the gastroesophageal junction on endoscopy, and presence of IM on biopsy evaluation (28). Currently, American Gastroenterological Association recommends screening for $\mathrm{BE}$ in patients with chronic GERD symptoms and multiple risk factors (i.e., 50 years of age or older, white race, male gender, obesity, history of smoking, family history for BE or EAC) (24).

\section{Issues in diagnosis of $B E$}

Endoscopic identification of salmon colored mucosa in the distal esophagus needs knowledge of landmarks for identification of the GEJ. These landmarks include the distal end of long palisading esophageal vessels and the proximal ends of gastric folds (29). These can be obscured in the presence of hiatal hernia or severe reflux esophagitis. ACG guidelines recommend adequate sampling in terms of procurement of at least four biopsy samples for every $2 \mathrm{~cm}$ segment of BE (24). Based on the length of columnar lined esophagus, $\mathrm{BE}$ can be short segment $\mathrm{BE}(>1 \mathrm{~cm}$ and $<3 \mathrm{~cm})$ and long segment $\mathrm{BE}(>3 \mathrm{~cm})$. The detection of intestinal metaplasia increased progressively with increasing length of abnormal columnar epithelium, being present in $70.4 \%$ in the $1-$ to $2-\mathrm{cm}$ group, $89.5 \%$ in the $3-$ to $4-\mathrm{cm}$ group, and $100 \%$ within the greater than or equal to $5 \mathrm{~cm}$ group (30). The number of biopsy samples is important as intestinal metaplasia can be missed on initial biopsy evaluation in short segment BE. Repeat endoscopy and biopsy is recommended in such situations (31). Since the definition of $\mathrm{BE}$ requires endoscopic identification of $\geq 1 \mathrm{~cm}$ length of columnar lined esophagus, it is recommended that, in absence of endoscopic findings, pathologists be descriptive in diagnosis of biopsies from GEJ or distal esophagus. They should describe the presence or absence of intestinal metaplasia. If intestinal metaplasia is present, this feature can be further qualified with a comment mentioning "IM seen in the biopsy may be representative of $B E$ if biopsies are derived from the tubular esophagus in the setting of appropriate endoscopic features" (29). This is because the intestinal metaplasia of the GEJ or gastric cardia is not associated with increased risk of cancer. Some histologic features such as presence of esophageal glands/ducts, multilayered epithelium and buried BE (Barrett's mucosa underlying intact squamous epithelium) suggest that biopsies are esophageal in origin (32).

Pseudogoblet cells are common mimickers of true goblet cells of BE. Intestinal metaplasia consists of singly lying true goblet-shaped cells interspersed randomly in foveolar epithelium. These cells contain acidic mucin (carbohydraterich proteins) which stain pale blue on routine hematoxylin and eosin stain, and intensely blue on Alcian blue at $\mathrm{pH}$ 2.5. In comparison, gastric nongoblet cell mucosa stain magenta/ red on PAS staining. Pseudogoblet cells are commonly seen in cardiac mucosa. These cells are also goblet-shaped surface mucous cells, which in contrast to true goblet cells, tend to cluster together. Interobserver agreement for diagnosing true goblet cells, and distinguishing them from pseudogoblet cells, has been shown to be extremely poor (33). PAS and alcian blue stain at $\mathrm{pH} 2.5$ stains pseudogoblet cells dark magenta in color as compared to true goblet cells. Some cases may show intense blue staining, similar to true goblet cells. Presence of columnar blue cells is a diagnostic pitfall of this histochemical stain. Columnar blue cells are mucinous columnar cells without the characteristic goblet shaped mucin droplet that stain blue with PAS with alcian blue at $\mathrm{pH}$ of 2.5 .

Multilayered epithelium (MLE) has been considered as a precursor to $\mathrm{BE}(32,34)$. This consists of 4-8 layers of basally located squamous cells and associated superficial columnar mucinous epithelium that can mimic goblet cells. Study by Glickman et al. 2009 showed that MLE from patients with GERD and patients with BE is immunophenotypically similar sharing expression of markers CDX 2 and MUC 2, thereby indicating that MLE represents early/transitional form of columnar metaplasia.

No definitive distinction can be made between the intestinal metaplasia arising from esophagus or gastric cardia. Multiple studies carried out a battery of immunohistochemical stains e.g., CK7, CK20, MUC1, 
MUC2, MUC6, HepPar1, MUC5AC, and DAS1, to differentiate $\mathrm{BE}$ from intestinal metaplasia of the cardiac mucosa (35-37), but all found similar immunophenotypic expression patterns among $\mathrm{BE}$ and cardiac mucosa with intestinal metaplasia.

\section{Dysplasia in BE}

Pathologist plays an important role in surveillance of $\mathrm{BE}$ by evaluating biopsies for features of dysplasia. Dysplasia is defined as neoplastic epithelium with cytologic and architectural atypia confined to the epithelium. The features evaluated include surface maturation, glandular architecture, cytologic atypia and presence of inflammation/ erosions. Based on consensus among experts dysplasia in $\mathrm{BE}$ are classified into 4 categories: Negative for dysplasia; indefinite for dysplasia; low-grade dysplasia and high-grade dysplasia (38).

Negative for dysplasia (NDS): The BE mucosa shows preserved surface maturation characterized by darkly staining nuclei with stratification at the base of glands as compared to the surface where nuclei are paler, maintain polarity and lack stratification. The cytologic atypia is limited to the basal portion of glands. Architecturally the glands are round and surrounded by abundant lamina propria. Inflammation, if present, may show reactive nuclear changes (Figure 2A).

Indefinite for dysplasia (IND): The BE mucosa in this category shows changes in deeper glands suggestive/ consistent with dysplasia, however, the surface maturation is preserved. In this category the cytologic atypia includes nuclear hyperchromasia, nuclear membrane irregularities and increased mitosis. Sometimes the nuclear atypia in presence of active inflammation may appear exaggerated enough to be designated as indefinite for dysplasia.

Low-grade dysplasia (LGD): The Barrett's mucosa shows loss of surface maturation and architectural distortion with glandular crowding, in absence of active inflammation. There is a sharp contrast between neoplastic and non-neoplastic mucosa. Nuclei in the surface mucosa show hyperchromasia, nuclear enlargement, stratification and mucin loss. Mitotic figures can be seen on the surface (Figure 2B).

High-grade dysplasia: The Barrett's mucosa shows loss of surface maturation (as in LGD) and glandular crowding. The nuclei show loss of polarity and are rounded, enlarged, hyperchromatic with inconspicuous nucleoli. Mitoses are frequent. Inflammation is less in comparison to the architectural and cytologic atypia. Presence of ulceration, active inflammation and/or prominent nucleoli are features indicative of reactive/reparative changes due to a benign process, or are concerning for an associated invasive carcinoma. Additional features suggestive of invasive adenocarcinoma on biopsies include cribriform glandular architecture, luminal necrotic debris, ulceration, neutrophils within dysplastic glands and pagetoid spread of neoplastic cells in the overlying squamous mucosa (Figure 2C).

\section{Variants of dysplasia}

Deep crypt dysplasia: Dysplasia with cytologic features of low-grade or high-grade dysplasia limited to the deep glands. Surface maturation is preserved. This is a rare occurrence and has been found to be associated with risk of progression due to presence of molecular alterations similar to conventional dysplasia (39).

Foveolar type dysplasia: This is usually not associated with intestinal metaplasia. The dysplastic foveolar glands show mild architectural changes and architectural crowding. The glands are lined by low cuboidal to columnar epithelium with eosinophilic to clear cytoplasm. The nuclei are round to oval and show prominent nucleoli. Typical and atypical mitoses are seen. The dysplastic foveolar glands are positive for MUC5AC, rarely for MUC6, and negative CDX2 and MUC2, unlike the classic adenomatous dysplasia. Dysplasia with 'hybrid' features of intestinal and foveolar dysplasia can occur.

\section{Intramucosal adenocarcinoma}

Intramucosal adenocarcinoma is defined by invasion of carcinoma into lamina propria but not beyond muscularis mucosae. The features of intramucosal adenocarcinoma are syncytial growth pattern with back-to-back glands, presence of single cells and small clusters within lamina propria (Figure 2D). Desmoplasia may not be present but if present, it is very subtle.

As per AGA recommendations, the surveillance of $\mathrm{BE}$ with repeat endoscopy and biopsy sampling is done every 3-5 years if the biopsy is negative for dysplasia, 6-12 months for a biopsy-proven low-grade dysplasia. Endoscopic ablation therapy/or a follow-up every 3 months is recommended for high-grade dysplasia.

\section{Esophageal adenocarcinoma}

The majority of esophageal adenocarcinomas arise in the distal esophagus. Uncommonly, it can arise more proximally in heterotopic gastric mucosa. The development of EAC appears to occur through progressive accumulation 

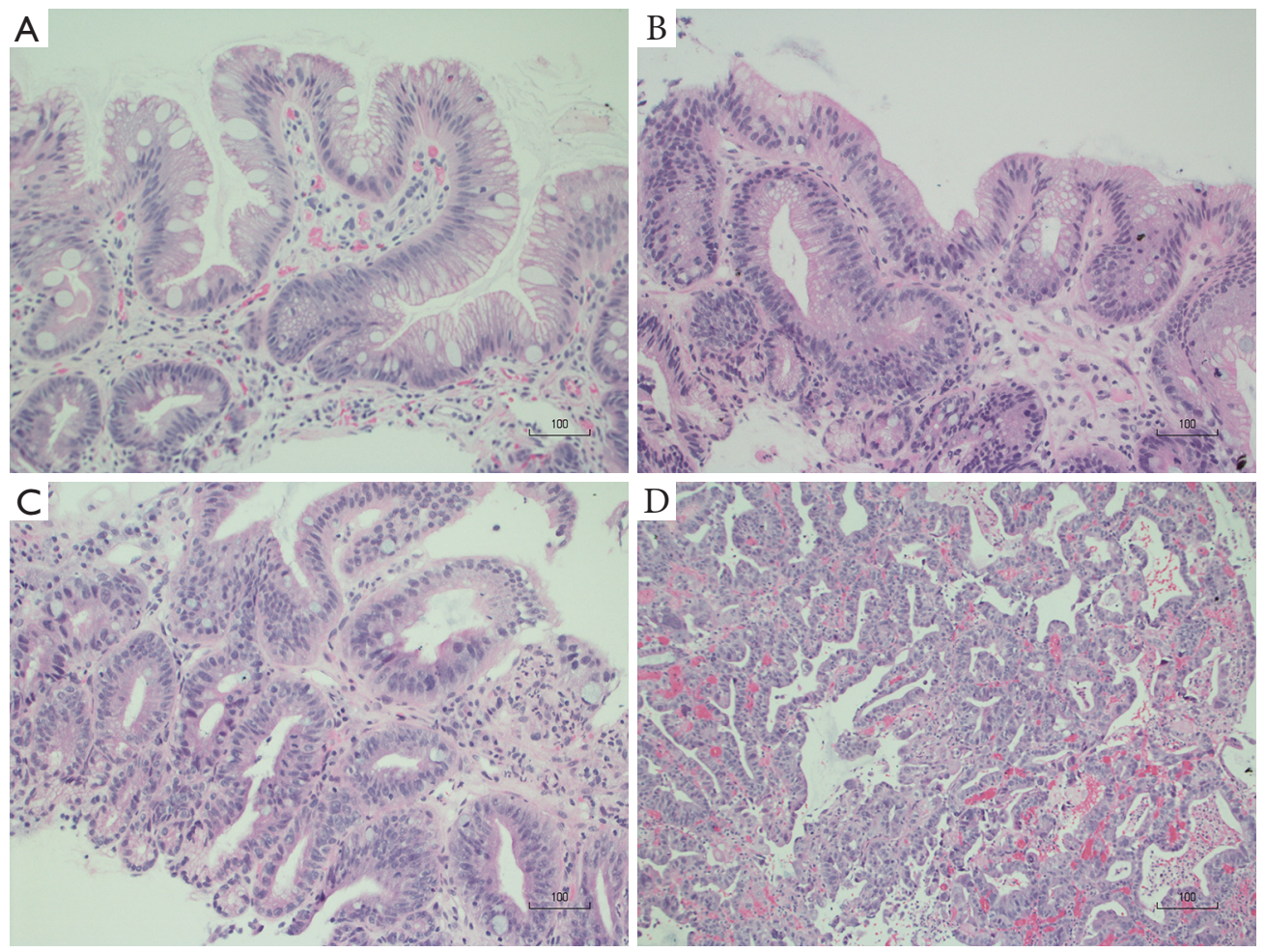

Figure 2 Barrett's esophagus dysplasia/neoplasia. (A) Barrett's esophagus, negative for dysplasia. Hematoxylin and Eosin stain $\times 200$; (B) Barrett's esophagus with low grade dysplasia. Hematoxylin and Eosin stain $\times 200$; (C) Barrett esophagus with high grade dysplasia. Hematoxylin and Eosin stain $\times 200$; (D) Barrett's esophagus with intramucosal adenocarcinoma. Hematoxylin and Eosin stain $\times 100$.

of multiple genetic abnormalities such as mutational inactivation of $\mathrm{p} 16$, and $\mathrm{p} 53$ genes, cell cycle abnormalities and aneuploidy (40). The molecular mechanism is complex and cannot be attributed to a single genetic event (41). Endoscopically, if detected early these tumors will present as mucosal irregularities. In later stages they appear as ulcerated/infiltrative or exophytic masses with obstruction. Histologically, these are gland-forming tumors with a tubular, tubulopapillary or papillary growth pattern. A small subset of cases shows mucinous differentiation. A few cases of diffuse signet ring cell adenocarcinoma have also been reported (42). Foci of BE with high-grade dysplasia are commonly seen in epithelium adjacent to the tumor. The tumors show variable grades of differentiation based on the amount of gland formation, and the nuclear atypia generally follows the grade of differentiation. Well-differentiated tumors show more than $95 \%$ gland formation, moderately differentiated tumors show $50-95 \%$ gland formation and poorly differentiated tumors show $<50 \%$ gland formation. Differential diagnosis includes metastasis or direct extension from tumors of lung or breast. Morphologically these mimic primary adenocarcinoma of esophagus. Immunohistochemical staining is helpful to differentiate. In contrast to primary adenocarcinoma of esophagus, adenocarcinoma of the lung is positive for immunostain for thyroid transcription factor 1 (TTF-1), and breast adenocarcinoma is positive for immunostains for estrogen receptor (ER) and GATA 3.

Rare tumors can be biphenotypic, with both squamous and mucinous/glandular differentiation. The tumors with intimate admixture of squamous and mucinous elements are designated as mucoepidermoid carcinoma. Tumors composed of two separate squamous and mucinous elements, adjacent to each other, are termed as adenosquamous carcinoma. Tumor stage at diagnosis determines prognosis for these biphenotypic tumors.

\section{Pathologic stage and prognostic factors of esophageal cancers}

The surgical resection specimens for esophageal cancer typically comprise of endoscopic mucosal resection (EMR), 

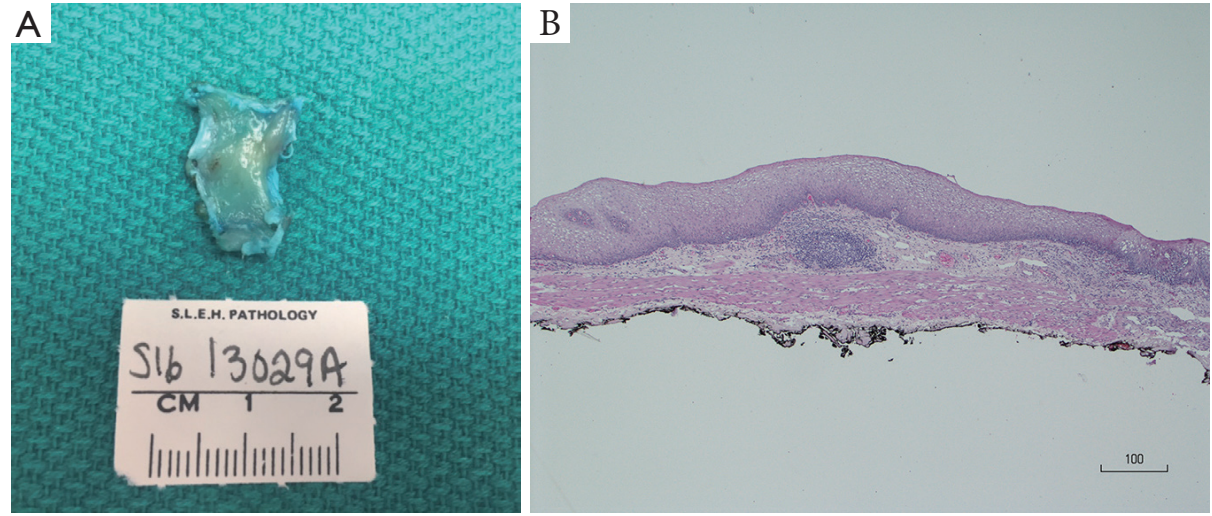

Figure 3 Endoscopic submucosal dissection for esophageal lesions. (A) Gross image of endoscopic submucosal dissection for squamous cell carcinoma-in-situ; (B) histologic section of tissue from endoscopic submucosal dissection. Hematoxylin and Eosin stain $\times 20$.

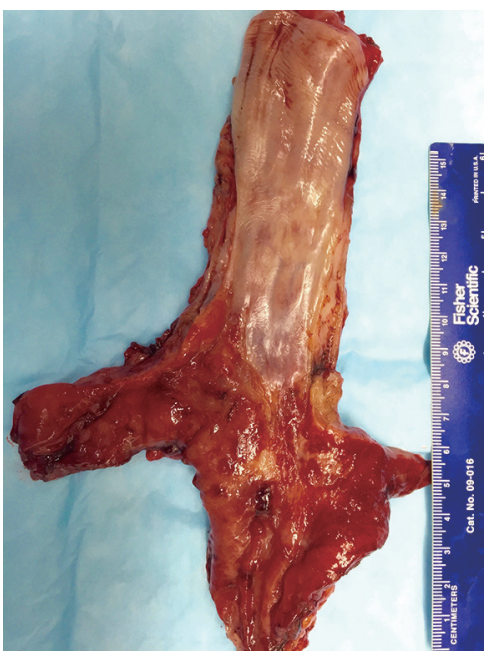

Figure 4 Gross image of distal esophagectomy, status-post neoadjuvant chemoradiation, with tumor at the gastroesophageal junction.

endoscopic submucosal dissection (ESD) (Figure 3) and/or esophagectomy with lymphadenectomy (Figure 4). Pathologic evaluation is performed to determine the presence/absence of residual tumor post-neoadjuvant chemoradiation, depth of invasion ( $\mathrm{pT}$ stage), tumor regression grade and nodal metastasis $(\mathrm{pN})$. The pathologic reporting is done on a standardized format jointly provided by the American Joint Committee on Cancer (AJCC)/Union for International Cancer Control (UICC), and College of American Pathologists (CAP). Currently the AJCC/UICC TNM $7^{\text {th }}$ edition (43) is the staging system used for esophageal resection specimens to predict prognosis in ESCC and
EAC (Table 1). Several studies have shown that assessment of pathologic response to neoadjuvant therapy, in terms of tumor regression grade and nodal metastasis, are independent prognostic factors for both EAC and ESCC (44-46).

\section{Esophageal squamous cell carcinoma}

Superficial squamous cell carcinoma is an early invasive esophageal cancer confined to mucosa or submucosa with/without lymph node metastasis (stage T1 by AJCC/ UICC TNM 7th edition). These have better prognosiscompared to deep/conventional ESCC with an overall 5 -year survival of greater than $60 \%$ (47). The superficial squamous carcinomas are further sub-classified based on depth of invasion: $\mathrm{m} 1$ intraepithelial non-invasive carcinoma, $\mathrm{m} 2$ carcinoma invading into lamina propria, m3 carcinoma extending to or invading muscularis mucosae, sm 1 carcinoma invading into superficial onethird submucosa, sm2 carcinoma invading into middle onethird submucosa and $\mathrm{sm} 3$ carcinoma invading into lower one-third of submucosa (48). Some studies have reported presence of lymph node metastasis in submucosal (T1b, sm1/2/3) tumors, but absence of lymph node metastasis in tumors with only mucosal invasion (stage $\mathrm{T} 1 \mathrm{a}$ including $\mathrm{m} 1, \mathrm{~m} 2$ and $\mathrm{m} 3$ ) (49). Others studies (50,51), have reported no lymph node metastasis in $\mathrm{m} 1$ lesions, but have found lymph node metastasis in a small percentage of $\mathrm{m} 1 / \mathrm{m} 2$ tumors, as well as in sm1/2/3 tumors. Endoscopic mucosal resection (EMR) or endoscopic submucosal dissection (ESD) is frequently used to treat early ESCC (Stage T1a). Surgery is currently the standard treatment for cancers with submucosal invasion (stage T1b) in ESCC (52). 


\begin{tabular}{|c|c|}
\hline Categories & Criteria \\
\hline \multicolumn{2}{|l|}{ pT category } \\
\hline pTX & Cannot be assessed \\
\hline pTO & No evidence of primary tumor \\
\hline pT1a & Tumor invades lamina propria or muscularis mucosae \\
\hline $\mathrm{pT} 1 \mathrm{~b}$ & Tumor invades submucosa \\
\hline pT2 & Tumor invades muscularis propria \\
\hline pT3 & Tumor invades adventitia \\
\hline \multicolumn{2}{|l|}{$\mathrm{pN}$ category } \\
\hline $\mathrm{pNX}$ & Cannot be assessed \\
\hline pNO & No regional lymph node metastasis \\
\hline pN1 & Regional lymph node metastasis involving 1-2 nodes \\
\hline $\mathrm{pN} 2$ & 3-6 nodes involved \\
\hline pN3 & 7 or more nodes involved \\
\hline \multicolumn{2}{|l|}{ pM category } \\
\hline
\end{tabular}

\section{Deep/conventional esophageal carcinoma}

Infiltration of squamous cell carcinoma into deeper layers of esophageal wall is common and is associated with poor prognosis due to the risk of lymph node metastasis and systemic metastasis. These usually present late and the standard treatment is neoadjuvant radiotherapy and/or chemotherapy. Surgery is usually done after neoadjuvant treatment. The overall survival of ESCC is $36.4 \%$ (53). Prognosis depends on the depth of mural invasion, tumor regression grade and lymph node metastasis. The tumornode-metastasis (TNM) system by the American Joint Committee on Cancer and the International Union Against Cancer 7 th Edition is the most widely used system for prognostication in treatment-naïve esophageal resections and for resections post neoadjuvant chemoradiation. For prognostic stage groupings, this system also incorporates the tumor location and histologic grade, besides the TNM stage. Tumor regression grade is not taken into account for determination of the stage groups in the current staging system. Wang et al. (53) validated the 7 th edition staging system for ESCC and reported significant differences in survival among different stage groups. They reported 5 -year stage-based survival rates as follows: IA, $84.9 \%$; IB, 70.9\%; IIA, 56.2\%; IIB, 43.3\%; IIIA, 37.9\%; IIIB, $23.3 \%$; IIIC, $12.9 \%$ and IV, $3.4 \%$. However, they did not find histologic grade or cancer location to be important/independent prognostic factors.

\section{Esophageal adenocarcinoma (EAC)}

These tumors involve the distal esophagus and the gastroesophageal junction (GEJ). Adenocarcinoma of the GEJ is a tumor whose epicenter is located within $5 \mathrm{~cm}$ of esophageal or gastric side of GEJ (54). Siewert classification is a widely used anatomic classification of adenocarcinoma of GEJ that is based on the location of the tumor with 
respect to the gastric cardia and has three types: Siewert type I tumors are adenocarcinoma of distal esophagus, Siewert type II tumors are adenocarcinoma of gastric cardia, and Siewert type III tumors are subcardial adenocarcinoma of proximal stomach infiltrating the GEJ (55). TMN pathologic stage is the most important prognostic factor for esophageal adenocarcinoma. Presence of nodal metastasis is related to the depth of tumor infiltration. Endoscopic resection, either EMR or ESD is the preferred treatment, as compared to esophagectomy, for early stage EAC because of low risk of nodal metastasis. This includes tumors with invasion into lamina propria and muscularis mucosae (stage 1a) and stage $1 \mathrm{~b}$ tumors with sm1 depth of invasion (49). Esophagectomy with standard lymphadenectomy is the treatment of choice for stage $1 \mathrm{~b}$ tumors with $\mathrm{sm} 2 / 3$ depth of invasion, because of increased risk of nodal metastasis (49). Presence of lymphovascular invasion and positive deep margin has been shown to be associated with reduced overall survival in T1 EAC (56). Number of positive lymph nodes also has an important prognostic factor (57). Survival in EAC decreases with increase in depth of invasion (pT), with nodal metastasis $(\mathrm{pN})$, positive resection margins and increasing histologic grades (58).

\section{HER 2 and gastroesophageal junction adenocarcinoma}

Human epidermal growth factor receptor (HER 2) is a tyrosine kinase receptor encoded by proto-oncogene HER2 (ERBB2) located on chromosome 17. This receptor belongs to the epidermal growth factor receptor family and its phosphorylation leads to cell division, proliferation, differentiation and apoptosis.

Drugs targeted against tumors expressing HER 2 protein have been found to have survival benefit in breast cancers. An international phase 3 randomized clinical trial using monoclonal anti HER 2 antibody, trastuzumab, directed against HER 2 protein was found to prolong survival in adenocarcinomas of stomach and gastroesophageal junction that expressed HER2 (59).

The HER 2 status of tumor can be assessed by immunohistochemistry on biopsies as well as resections. In-situ hybridization (ISH) is used to further confirm the equivocal (score 2) cases on immunohistochemistry. Based on the guidelines provided by National comprehensive cancer network (NCCN), trastuzumab is given only to patients with advanced GEJ/gastric adenocarcinoma showing positive staining (score 3 ) for HER2 by immunohistochemistry, and with evidence of HER2 amplification by ISH in cases, which showed equivocal staining (score 2) by IHC (60).

\section{Acknowledgements}

None.

\section{Footnote}

Conflicts of Interest: The authors have no conflicts of interest to declare.

\section{References}

1. SEER Cancer Statistics Factsheets: Esophageal Cancer. National Cancer Institute. Bethesda, MD. Available online: http://seer.cancer.gov/statfacts/html/esoph.html

2. Zhang Y. Epidemiology of esophageal cancer. World J Gastroenterol 2013;19:5598-606.

3. Thrift AP, Whiteman DC. The incidence of esophageal adenocarcinoma continues to rise: analysis of period and birth cohort effects on recent trends. Ann Oncol 2012;23:3155-62.

4. Morita M, Kumashiro R, Kubo N, et al. Alcohol drinking, cigarette smoking, and the development of squamous cell carcinoma of the esophagus: epidemiology, clinical findings, and prevention. Int J Clin Oncol 2010;15:126-34.

5. Akhtar S, Sheikh AA, Qureshi HU. Chewing areca nut, betel quid, oral snuff, cigarette smoking and the risk of oesophageal squamous-cell carcinoma in SouthAsians: a multicentre case-control study. Eur J Cancer 2012;48:655-61.

6. Alsop BR. Sharma P. Esophageal Cancer. Gastroenterol Clin North Am 2016;45:399-412.

7. Bucchi D, Stracci F, Buonora N, et al. Human papillomavirus and gastrointestinal cancer: A review. World J Gastroenterol 2016;22:7415-30.

8. Ellis A, Risk JM, Maruthappu T, et al. Tylosis with oesophageal cancer: Diagnosis, management and molecular mechanisms. Orphanet J Rare Dis 2015;10:126.

9. Rios-Galvez S, Meixueiro-Daza A, Remes-Troche JM. Achalasia: a risk factor that must not be forgotten for esophageal squamous cell carcinoma. BMJ Case Rep 2015;2015. pii: bcr2014204418.

10. Chang-Claude JC, Wahrendorf J, Liang QS, et al. An epidemiological study of precursor lesions of esophageal cancer among young persons in a high-risk population in 
Huixian, China. Cancer Res 1990;50:2268-74.

11. Qiu SL, Yang GR. Precursor lesions of esophageal cancer in high-risk populations in Henan Province, China. Cancer 1988;62:551-7.

12. Dawsey SM, Lewin KJ, Wang GQ, et al. Squamous esophageal histology and subsequent risk of squamous cell carcinoma of the esophagus. A prospective follow-up study from Linxian, China. Cancer 1994;74:1686-92.

13. Shimizu M, Nagata K, Yamaguchi H, et al. Squamous intraepithelial neoplasia of the esophagus: past, present, and future. J Gastroenterol 2009;44:103-12.

14. Mori M, Adachi Y, Matsushima T, et al. Lugol staining pattern and histology of esophageal lesions. Am J Gastroenterol 1993;88:701-5.

15. Sepehr A, Razavi P, Saidi F, et al. Esophageal exfoliative cytology samplers. A comparison of three types. Acta Cytol 2000;44:797-804.

16. Tajima Y, Nakanishi Y, Tachimori Y, et al. Significance of involvement by squamous cell carcinoma of the ducts of esophageal submucosal glands. Analysis of 201 surgically resected superficial squamous cell carcinomas. Cancer 2000;89:248-54.

17. Chu P, Stagias J, West AB, et al. Diffuse pagetoid squamous cell carcinoma in situ of the esophagus: a case report. Cancer 1997;79:1865-70.

18. Pesko P, Rakic S, Milicevic M, et al. Prevalence and clinicopathologic features of multiple squamous cell carcinoma of the esophagus. Cancer 1994;73:2687-90.

19. Wang GQ, Abnet CC, Shen Q, et al. Histological precursors of oesophageal squamous cell carcinoma: results from a 13 year prospective follow up study in a high risk population. Gut 2005;54:187-92.

20. Raza MA, Mazzara PF. Sarcomatoid carcinoma of esophagus. Arch Pathol Lab Med 2011;135:945-8.

21. Cavallin F, Scarpa M, Alfieri R, et al. Esophageal carcinosarcoma: management and prognosis at a single Italian series. Anticancer Res 2014;34:7455-9.

22. Mansour NM, Groth SS, Anandasabapathy S. Esophageal Adenocarcinoma: Screening, Surveillance, and Management. Annu Rev Med 2017;68:213-227.

23. Schneider JL, Corley DA. A review of the epidemiology of Barrett's oesophagus and oesophageal adenocarcinoma. Best Pract Res Clin Gastroenterol 2015;29:29-39.

24. American Gastroenterological Association, Spechler SJ, Sharma P, et al. American Gastroenterological Association medical position statement on the management of Barrett's esophagus. Gastroenterology 2011;140:1084-91.

25. Fitzgerald RC, di Pietro M, Ragunath K, et al.
British Society of Gastroenterology. British Society of Gastroenterology guidelines on the diagnosis and management of Barrett's oesophagus. Gut 2014;63:7-42.

26. Sampliner RE. Practice guidelines on the diagnosis, surveillance, and therapy of Barrett's esophagus. The Practice Parameters Committee of the American College of Gastroenterology. Am J Gastroenterol 1998;93:1028-32.

27. Bhat S, Coleman HG, Yousef F, et al. Risk of malignant progression in Barrett's esophagus patients: results from a large population-based study. J Natl Cancer Inst 2011;103:1049-57.

28. Shaheen NJ, Falk GW, Iyer PG, et al. Corrigendum: ACG Clinical Guideline: Diagnosis and Management of Barrett's Esophagus. Am J Gastroenterol 2016;111:1077.

29. Naini BV, Chak A, Ali MA, et al. Barrett's oesophagus diagnostic criteria: endoscopy and histology. Best Pract Res Clin Gastroenterol 2015;29:77-96.

30. Chandrasoma PT, Der R, Ma Y, et al. Histologic classification of patients based on mapping biopsies of the gastroesophageal junction. Am J Surg Pathol 2003;27:929-36.

31. Jones TF, Sharma P, Daaboul B, et al. Yield of intestinal metaplasia in patients with suspected short-segment Barrett's esophagus (SSBE) on repeat endoscopy. Dig Dis Sci 2002;47:2108-11.

32. Srivastava A, Odze RD, Lauwers GY, et al. Morphologic features are useful in distinguishing Barrett's esophagus from carditis with intestinal metaplasia. Am J Surg Pathol 2007;31:1733-41.

33. Wang H, Brown I, Kumarasinghe P, et al. Poor agreement for detection of goblet cells in esophageal and GEJ biopsies. Lab Invest 2012;92:184A-185A.

34. Soucy G, Onstad L, Vaughan TL, et al. Histologic Features Associated With Columnar-lined Esophagus in Distal Esophageal and Gastroesophageal Junction (GEJ) Biopsies From GERD Patients: A Community-based Population Study. Am J Surg Pathol 2016;40:827-35.

35. DeMeester SR, Wickramasinghe KS, Lord RV, et al. Cytokeratin and DAS-1 immunostaining reveal similarities among cardiac mucosa, CIM, and Barrett's esophagus. Am J Gastroenterol 2002;97:2514-23.

36. Chu PG, Jiang Z, Weiss LM. Hepatocyte antigen as a marker of intestinal metaplasia. Am J Surg Pathol 2003;27:952-9.

37. Gulmann C, Shaqaqi OA, Grace A, et al. Cytokeratin 7/20 and MUC1, 2, 5AC, and 6 expression patterns in Barrett's esophagus and intestinal metaplasia of the stomach: intestinal metaplasia of the cardia is related to Barrett's 
esophagus. Appl Immunohistochem Mol Morphol 2004;12:142-7.

38. Montgomery E, Bronner MP, Goldblum JR, et al. Reproducibility of the diagnosis of dysplasia in Barrett's esophagus: a reaffirmation. Hum Pathol 2001;32:368-78.

39. Lomo LC, Blount PL, Sanchez CA, et al. Crypt dysplasia with surface maturation: a clinical, pathologic, and molecular study of a Barrett's esophagus cohort. Am J Surg Pathol 2006;30:423-35.

40. Wijnhoven BP, Tilanus HW, Dinjens WN. Molecular biology of Barrett's adenocarcinoma. Ann Surg 2001;233:322-37.

41. Kalatskaya I. Overview of major molecular alterations during progression from Barrett's esophagus to esophageal adenocarcinoma. Ann N Y Acad Sci 2016;1381:74-91.

42. Maezato K, Nishimaki T, Oshiro M, et al. Signet-ring cell carcinoma of the esophagus associated with Barrett's epithelium: report of a case. Surg Today 2007;37:1096-101.

43. Edge SB, Byrd DR, Compton CC, et al. editors. American Joint Committee on Cancer Staging Manual. 7th ed. New York: Springer-Verlag, 2010.

44. Wu TT, Chirieac LR, Abraham SC, et al. Excellent interobserver agreement on grading the extent of residual carcinoma after preoperative chemoradiation in esophageal and esophagogastric junction carcinoma: a reliable predictor for patient outcome. Am J Surg Pathol 2007;31:58-64.

45. Donohoe CL, O'Farrell NJ, Grant T, et al. Classification of pathologic response to neoadjuvant therapy in esophageal and junctional cancer: assessment of existing measures and proposal of a novel 3-point standard. Ann Surg 2013;258:784-92; discussion 792.

46. Karamitopoulou E, Thies S, Zlobec I, et al. Assessment of tumor regression of esophageal adenocarcinomas after neoadjuvant chemotherapy: comparison of 2 commonly used scoring approaches. Am J Surg Pathol 2014;38:1551-6.

47. Igaki H, Kato H, Tachimori Y, et al. Prognostic evaluation of patients with clinical T1 and T2 squamous cell carcinomas of the thoracic esophagus after 3-field lymph node dissection. Surgery 2003;133:368-74.

48. Shimizu M, Zaninotto G, Nagata K, et al. Esophageal squamous cell carcinoma with special reference to its early stage. Best Pract Res Clin Gastroenterol 2013;27:171-86.

49. Ancona E, Rampado S, Cassaro M, et al. Prediction of lymph node status in superficial esophageal carcinoma. Ann Surg Oncol 2008;15:3278-88.

50. Eguchi T, Nakanishi Y, Shimoda T, et al. Histopathological criteria for additional treatment after endoscopic mucosal resection for esophageal cancer: analysis of 464 surgically resected cases. Mod Pathol 2006;19:475-80.

51. Zhou Y, Du J, Li H, et al. Clinicopathologic analysis of lymph node status in superficial esophageal squamous carcinoma. World J Surg Oncol 2016;14:259.

52. Sgourakis G, Gockel I, Lang H. Endoscopic and surgical resection of T1a/T1b esophageal neoplasms: A systematic review. World J Gastroenterol 2013;19:1424-37.

53. Wang J, Wu N, Zheng QF, et al. Evaluation of the 7th edition of the TNM classification in patients with resected esophageal squamous cell carcinoma. World J Gastroenterol 2014;20:18397-403.

54. Mullen JT, Kwak EL, Hong TS. What's the Best Way to Treat GE Junction Tumors? Approach Like Gastric Cancer. Ann Surg Oncol 2016;23:3780-5.

55. Siewert JR, Stein HJ. Classification of adenocarcinoma of the oesophagogastric junction. Br J Surg 1998;85:1457-9.

56. Leggett CL, Lewis JT, Wu TT, et al. Clinical and histologic determinants of mortality for patients with Barrett's esophagus-related T1 esophageal adenocarcinoma. Clin Gastroenterol Hepatol 2015;13:65864.e1-3.

57. Hofstetter W, Correa AM, Bekele N, et al. Proposed modification of nodal status in AJCC esophageal cancer staging system. Ann Thorac Surg 2007;84:365-73; discussion 374-5.

58. Rice TW, Apperson-Hansen C, DiPaola LM, et al. Worldwide Esophageal Cancer Collaboration: clinical staging data. Dis Esophagus 2016;29:707-14.

59. Bang YJ, Van Cutsem E, Feyereislova A, et al. Trastuzumab in combination with chemotherapy versus chemotherapy alone for treatment of HER2-positive advanced gastric or gastro-oesophageal junction cancer (ToGA): a phase 3, open-label, randomised controlled trial. Lancet 2010;376:687-97.

60. Bartley AN, Christ J, Fitzgibbons PL, et al. Template for Reporting Results of HER2 (ERBB2) Biomarker Testing of Specimens From Patients With Adenocarcinoma of the Stomach or Esophagogastric Junction. Arch Pathol Lab Med 2015;139:618-20.

Cite this article as: Jain S, Dhingra S. Pathology of esophageal cancer and Barrett's esophagus. Ann Cardiothorac Surg 2017;6(2):99-109. doi: 10.21037/acs.2017.03.06 